\title{
Directed transport in a ratchet with internal and chemical freedoms
}

\author{
T. Dittrich ${ }^{\mathrm{a}, \mathrm{b}}$, N. A. Naranjo ${ }^{\mathrm{a}, \mathrm{b}}$ \\ ${ }^{a}$ Depto. de Física, Universidad Nacional de Colmbia, Bogotá D.C., Colombia \\ ${ }^{b}$ CeiBA-Complejidad, Bogotá D.C., Colombia
}

\begin{abstract}
We consider mechanisms of directed transport in a ratchet model comprising, besides the external freedom where transport occurs, a chemical freedom that replaces the familiar external driving by an autonomous dynamics providing energy input, and an internal freedom representing a functional mode of a motor molecule. The dependence of the current on various parameters is studied in numerical simulations of our model. In particular, we point out the rôle of the internal freedom as a buffer between energy input and output of mechanical work that allows a temporary storage of injected energy and can contribute to the efficiency of current generation.
\end{abstract}

Key words: ratchet, directed transport, internal freedom, molecular motor PACS: 05.45.Ac, 36.20.Ey, 87.15.hp

\section{Introduction}

Research on directed transport at the microscopic level has benefitted enormously from the interaction and mutual stimulation of applied studies close to experimentally accessible phenomena and theoretical analyses, the paradigm case being the concept of Brownian motors 1, 2, 3, 4, as it emerged from the discovery of motor molecules generating directed transport in the living cell [5, 6. Notwithstanding, the distance between the two endeavours remains large. An example is the degree of structural complexity, reflected in the number of degrees of freedom involved, of the models considered on either side: While realistic molecular-dynamics simulations of biomolecules typically include the full set of hundreds or thousands of nuclear freedoms, plus possibly some of the surrounding solvent (for a review see, e.g., 77), the key notion of ratchets has been developed considering mere point particles in one-dimensional periodic potentials $[8,9$.

Efforts are under way on both sides to bring these ends closer to one another. On the one hand, in molecular biophysics, the concept of functional degree of freedom [10, 11] has been conceived for largescale conformational changes on long timescales that are immediately responsible, e.g., for the catalytic activity of a protein, and can be identified on basis of objective criteria such as normal-mode Preprint submitted to Chemical Physics analysis [12, 13, 14] to establish a hierarchy among the modes from slow to fast and from collective (global) to microscopic. On the other hand, elementary ratchet models are being endowed one by one with additional freedoms to study their possible rôle in directed transport [15].

The present work is intended to insert another stepstone from the side of abstract ratchet models towards biophysical realism, by incorporating a few crucial features of molecular motors: We devise a model that includes an internal freedom to represent some functionally relevant conformational change. The concept of molecular combustion motor is incorporated replacing the familiar periodic external driving by a chemical freedom as coherent energy source which maintains the system far from thermal equilibrium [16]. The ubiquitous rôle of thermal fluctuations is modeled, as usually, by random forces. At the same time, we avoid the detailed reconstruction of any specific system such as $\mathrm{F}_{1}$-ATPase [17, 18, 19] or even their reduction to "mechanical toy models" 20] which typically still are tailored to represent a certain (species of) motor molecule and therefore involve some contingency. This allows us to study the effect of internal freedoms on general grounds, applicable to a broad class of molecules and even beyond the biophysical realm. In this way, we take up themes set by pio-

July 28, 2021 
neering works such as Kaneko et al. [21] on molecular machines with internal freedoms and of Mateos on ratchets based on non-point particles [15]. At the same time, focussing on the biological context, we leave aside quantum effects which probably are of minor relevance for transport in the living cell, yet may be crucial in artificial nanodevices 22 .

The dynamics of our model is simulated in the underdamped regime, allowing for complex deterministic motion, and in the presence of thermal noise, analyzed as to transport mechanisms involving the internal freedom as essential element. We find evidence, above all, that it serves as a temporary energy storage which partially decouples directed transport from the energy source and thus decorrelates the discrete steps in the external freedom from the likewise approximately quantized discharges of chemical energy into the systemcertainly desirable features from the point of view of robustness, efficiency, and versatility of a molecular motor. Preliminary results of this work have been published (in Spanish) in Ref. 23.

Our model is motivated and constructed in Sec. 2 . Section 3 provides a survey of its dynamical behaviour in different parameter regimes. Transport properties and the rôle of the internal freedom are discussed in Sec. 4. We conclude in Sec. 5 with some remarks on open ends.

\section{Model}

We pretend to construct a minimal model of a molecular combustion motor that goes beyond the familiar ratchet scheme in two essential points, (i) it includes an internal freedom to represent a functional (slow, conformational) mode of the molecule, and (ii) it incorporates the injection of chemical energy, e.g., through hydrolysis of ATP, as autonomous dynamics of a chemical degree of freedom corresponding to the reaction coordinate underlying that process [16]. The formulation of our model is inspired in various respects by the example of F1ATPase, a prototypical rotational molecular motor 17, 18, 19, but does not intend its reconstruction in any biophysical detail, i.e., is to be considered an impressionist view, at most, of that molecule.

More precisely, we require the model to comply with the following conditions:

i. External freedom The coordinate $x_{\mathrm{ex}}$ is equivalent to the single freedom of the usual pointparticle ratchets. It will be subjected to a standard ratchet potential, periodic with period $X_{\mathrm{ex}}=2 \pi$ but breaking invariance under parity $x_{\mathrm{ex}} \rightarrow-x_{\mathrm{ex}}$, which can be considered either as cyclic (angle) or as extended. To model the conversion of internal kinetic energy into directed transport, it will be coupled to the internal freedom, but also to the chemical freedom from which it receives coherent energy.

ii. Internal freedom Following Kaneko et al. [21, we model the internal freedom $x_{\text {in }}$ like a pendulum, with a smooth periodic potential, period $X_{\text {in }}=2 \pi$, which however lacks the intentional asymmetry of the ratchet potential proper.

iii. Chemical freedom In order to reproduce the entropical bias of the "combustion" reaction, we impose a non-zero mean gradient on the otherwise periodic dependence of the potential on the chemical coordinate $x_{\mathrm{ch}}$, i.e., $V\left(x_{\mathrm{ex}}, x_{\mathrm{in}}, x_{\mathrm{ch}}+X_{\mathrm{ch}}\right)=V\left(x_{\mathrm{ex}}, x_{\mathrm{in}}, x_{\mathrm{ch}}\right)+E_{\mathrm{ch}}$, $E_{\text {ch }}$ denoting the net energy gain of the (hydrolysis etc.) reaction per molecule. To keep the behaviour of this coordinate close to an approximately periodic energy injection, even in presence of the backaction from the external freedom, we further choose a large inertia $m_{\mathrm{ch}}$.

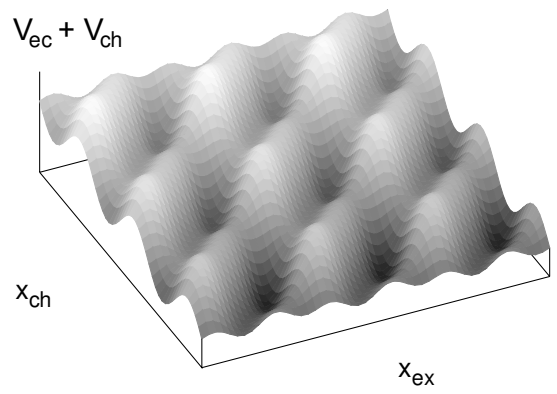

Figure 1: Potential (1) as a function of coordinates $x_{\mathrm{ex}}$ and $x_{\mathrm{ch}}$ for the external and the chemical freedoms, resp. Greylevel code ranges from black (low) to white (high).

To implement these requirements, we compose the full potential as follows:

$$
\begin{aligned}
V\left(x_{\mathrm{ex}}, x_{\mathrm{in}}, x_{\mathrm{ch}}\right)= & V_{\mathrm{ei}}\left(x_{\mathrm{ex}}, x_{\mathrm{in}}\right)+V_{\mathrm{in}}\left(x_{\mathrm{in}}\right)+ \\
& +V_{\mathrm{ec}}\left(x_{\mathrm{ex}}, x_{\mathrm{ch}}\right)+V_{\mathrm{ch}}\left(x_{\mathrm{ch}}\right) .
\end{aligned}
$$


where

$$
\begin{aligned}
V_{\mathrm{ei}}\left(x_{\mathrm{ex}}, x_{\mathrm{in}}\right)= & -\frac{1+\varepsilon_{\mathrm{ei}} \sin \left(x_{\mathrm{in}}\right)}{1+\varepsilon_{\mathrm{ei}}} \\
& \times \frac{\sin \left(x_{\mathrm{ex}}\right)+A \sin \left(2 x_{\mathrm{ex}}\right)}{2 f_{A}}, \\
V_{\mathrm{in}}\left(x_{\mathrm{in}}\right)= & -K \sin \left(x_{\mathrm{in}}\right), \\
V_{\mathrm{ec}}\left(x_{\mathrm{ex}}, x_{\mathrm{ch}}\right)= & -\varepsilon_{\mathrm{ec}} \sin \left(x_{\mathrm{ch}}\right) \sin \left(x_{\mathrm{ex}}-\delta\right), \\
V_{\mathrm{ch}}\left(x_{\mathrm{ch}}\right)= & -\frac{E_{\mathrm{ch}}}{2 \pi} x_{\mathrm{ch}} .
\end{aligned}
$$

The parameter $A$ determines the asymmetry of the ratchet potential [24]. The coupling between external and internal and between external and chemical freedoms is controlled, respectively, by $\varepsilon_{\mathrm{ei}}$ and $\varepsilon_{\mathrm{ec}}$. We plot the potential (1) as a function of external and chemical coordinates in Fig. 1. Diagonal channels corresponding to a rigid association of one hydrolysis reaction per spatial step in $x_{\text {ex }}$ can be discerned (cf. Ref. [16]).

Moreover, to represent the fast internal as well as ambient degrees of freedom in a collective manner, we include dissipation terms for $x_{\mathrm{ex}}$ and $x_{\mathrm{ch}}$ corresponding to velocity-proportional (Ohmic) friction. Working on the microscopic level, we complement them by random forces representing thermal noise, with autocorrelation functions related to dissipation rates through the fluctuation-dissipation theorem [25]. All in all, we thus arrive at a set of coupled stochastic differential equations [26],

$$
\begin{aligned}
m_{\mathrm{ex}} \ddot{x}_{\mathrm{ex}}= & -m_{\mathrm{ex}} \gamma_{\mathrm{ex}} \dot{x}_{\mathrm{ex}}+\frac{1+\varepsilon_{\mathrm{ei}} \sin \left(x_{\mathrm{in}}\right)}{1+\varepsilon_{\mathrm{ei}}} \\
& \times \frac{\cos \left(x_{\mathrm{ex}}\right)+2 A \cos \left(2 x_{\mathrm{ex}}\right)}{2 f_{A}} \\
& +\varepsilon_{\mathrm{ec}} \sin \left(x_{\mathrm{ch}}\right) \cos \left(x_{\mathrm{ex}}-\delta\right)+\xi(t), \\
m_{\mathrm{in}} \ddot{x}_{\mathrm{in}}= & K \cos \left(x_{\mathrm{in}}\right) \\
& +\frac{\varepsilon_{\mathrm{ei}} \cos \left(x_{\mathrm{in}}\right)}{1+\varepsilon_{\mathrm{ei}}} \frac{\sin \left(x_{\mathrm{ex}}\right)+A \sin \left(2 x_{\mathrm{ex}}\right)}{2 f_{A}}, \\
m_{\mathrm{ch}} \ddot{x}_{\mathrm{ch}}= & -m_{\mathrm{ch}} \gamma_{\mathrm{ch}} \dot{x}_{\mathrm{ch}}+\frac{\Delta V}{2 \pi} \\
& +\varepsilon_{\mathrm{ec}} \cos \left(x_{\mathrm{ch}}\right) \sin \left(x_{\mathrm{ex}}-\delta\right) .
\end{aligned}
$$

The random force $\xi(t)$ is defined by $\langle\xi(t)\rangle=0$ and $\langle\xi(t) \xi(0)\rangle=2 m_{\mathrm{ex}} \gamma_{\mathrm{ex}} k T \delta(t)$ at temperature $T$. The parameters $\gamma_{\text {ex }}$ and $\gamma_{\mathrm{ch}}$ are the friction coefficients for the external and the chemical freedoms, respectively.

The numerical results presented in the following have been obtained by solving Eqs. (6) by Conventional Brownian Dynamics, see Refs. [26, 27, 28.
Typical parameter values used in our simulations are summarized in Tab. 1

\begin{tabular}{lllllll}
\hline Parameter & $m_{\mathrm{ex}}$ & $m_{\mathrm{in}}$ & $m_{\mathrm{ch}}$ & $K$ & $\varepsilon_{\mathrm{ec}}$ & $\varepsilon_{\mathrm{ei}}$ \\
\hline Value & 1 & 0.1 & 100 & 0.1 & 1.0 & 1.0 \\
\hline \hline Parameter & $A$ & $k T$ & $\Delta V$ & $\delta$ & $\gamma_{\mathrm{ex}}$ & $\gamma_{\mathrm{ch}}$ \\
\hline Value & 0.25 & 1 & 100 & 4.53 & 0.1 & 1 \\
\hline
\end{tabular}

Table 1: Default values for the parameters of our model used wherever not indicated otherwise.

\section{Dynamics and phase-space structure}

The principal parameters determining the behaviour of our model are $A$ (asymmetry of the ratchet potential), $\varepsilon_{\mathrm{ei}}$ (coupling external to internal freedom), $\varepsilon_{\mathrm{ec}}$ (coupling external to chemical freedom), $\gamma_{\text {ex }}, \gamma_{\text {ch }}$ (friction) and temperature $T$. This quite high-dimensional parameter space is delimited by the following asymptotics:

i. Symmetric external potential For $A \rightarrow 0$, the simultaneous presence of symmetry-related counterpropagating trajectory pairs identically cancels transport.

ii. Internal freedom decoupled In the case $\varepsilon_{\mathrm{ei}}=0$, the system reduces to a point-particle ratchet, broadly documented in the literature in all its facets (overdamped, underdamped with nontrivial dynamics beyond mere relaxation, Hamiltonian), see 9 .

iii. Chemical freedom decoupled As we are dealing with an autonomous system without external driving, decoupling the chemical freedom deprives the ratchet of its energy source, so that transport dies out. Even in the Hamiltonian case, a driving is necessary for directed transport to occur, since there is then no other means to break time-reversal invariance (apart from magnetic fields which however play no rôle in a biochemical context).

iv. Hamiltonian dynamics In the limit $\gamma_{\mathrm{ex}}, \gamma_{\mathrm{ch}} \rightarrow$ 0 of vanishing friction, the system becomes a Hamiltonian ratchet with internal freedom. However, in the absence of an asymmetric external driving or a magnetic field, the system is then time-reversal invariant and directed currents are excluded. 
In the following, we consider in more detail a crucial aspect of the dynamics, the structure of the attractor and its connectivity along the lattice as a function of the "driving force", i.e., the coupling to the chemical freedom, as well as friction and noise strength.
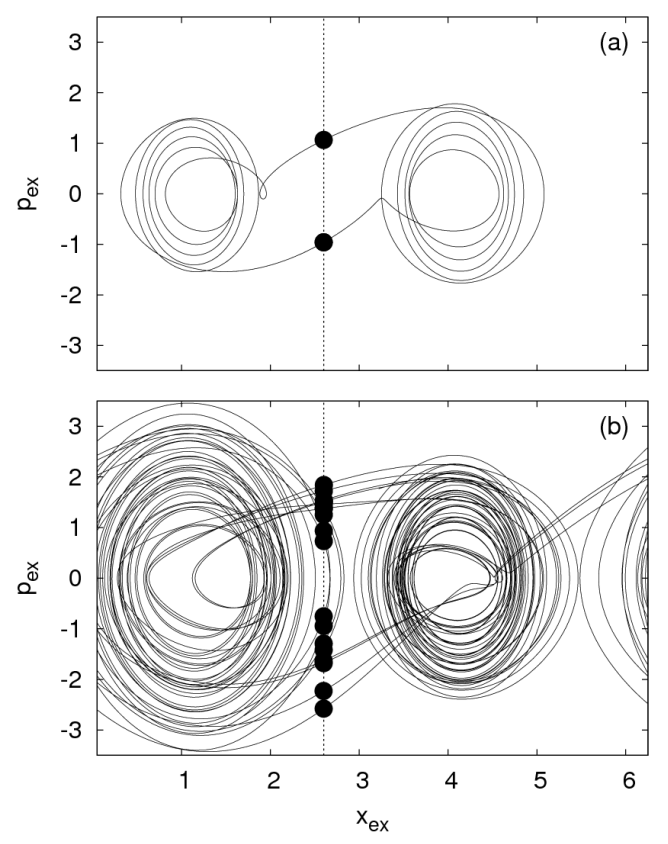

Figure 2: Attractor of the system 6 projected onto the plane $\left(p_{\text {ex }}, x_{\text {ex }}\right)$, for $\varepsilon_{\text {ec }}=1.0$ (a) and $7.1(\mathrm{~b})$. The dotted line indicates the surface of section underlying Fig. 7 below.

Figure 2 shows the attractor, as a converged trajectory projected onto the $\left(p_{\mathrm{ex}}, x_{\mathrm{ex}}\right)$-plane, for $\varepsilon_{\mathrm{ec}}=1.0$ (panel a) and $\varepsilon_{\mathrm{ec}}=7.1$ (b). While for low coupling we discern a limit cycle, restricted to a unit cell of the potential, for strong coupling it has given way to a strange attractor which connects across the unit cell boundaries along the entire lattice.

These two crucial "crises", though, the emergence of a strange attractor and its change of topology from isolated to conected, are independent of one another. This is evidenced in Fig. 3 a, analogous to Fig. 2 but at intermediate values of the coupling, where we observe a strange attractor still bounded within a unit cell. Conversely, Fig. 3b shows a limit cycle extending periodically along the lattice.

Besides the fundamental rôle of thermal fluctuations in directed transport through the "rectification of noise" outside equilibrium, it affects the dy-
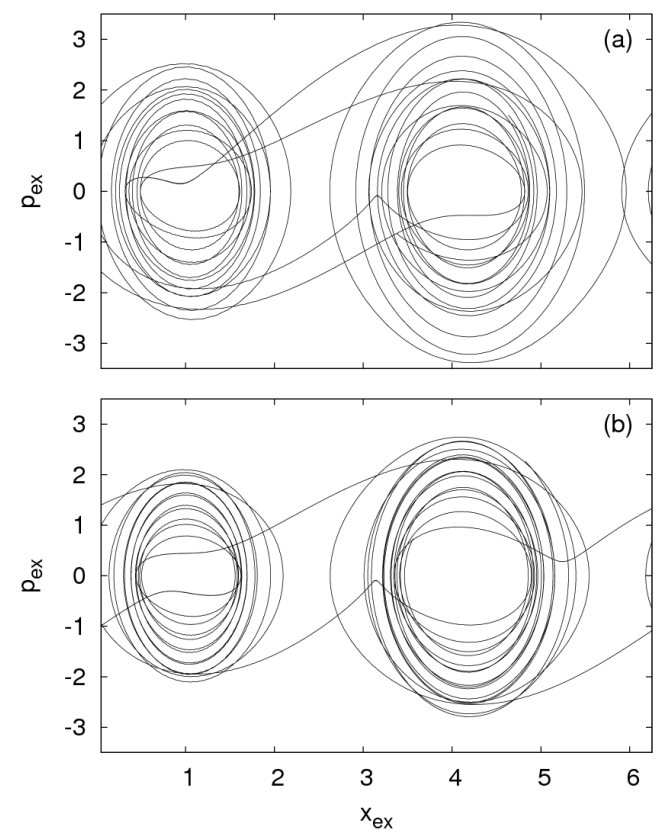

Figure 3: As Fig. 2 but for $\varepsilon_{\mathrm{ec}}=6.0$ (a) and 2.5 (b).

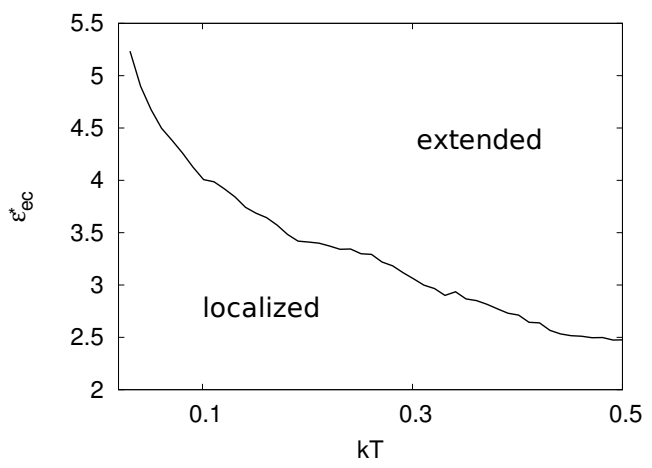

Figure 4: Threshold value of $\varepsilon_{\mathrm{ec}}^{*}$ for the transition from localized to extended attractors as a function of the bath temperature $T$.

namics in a more common way by softening attractor structures and thus contributing to a merger of attractors along the lattice. Figure 4 clearly demonstrates how noise tends to lower the threshold $\varepsilon_{\mathrm{ec}}^{*}$ in terms of the coupling to the chemical freedom for the transition to transport along the system. 


\section{Directed transport}

We here measure directed transport in terms of the mean velocity of the external freedom,

$$
I=\left\langle\dot{x}_{\mathrm{ex}}\right\rangle=\lim _{t \rightarrow \infty} \frac{1}{t}\left\langle x_{\mathrm{ex}}(t)-x_{\mathrm{ex}}(0)\right\rangle
$$

taking averages, e.g., over initial conditions within the same basin of attraction or over realizations of a stochastic force. The generation of non-zero currents in ratchet-like systems depends on the following independent general necessary conditions:

i. The breaking of all binary symmetries involving an inversion of momentum, such as in particular time reversal and parity [24].

ii. The existence of trajectories extending over different unit cells of the potential, or, if the external freedom is cyclic, corresponding to rotational as opposed to librational motion.

Concerning condition (i), in the absence of an external driving or a coupling to a magnetic field, it is only the friction terms in the equations of motion which break time-reversal invariance. It is evident from Fig. 5 that there is no transport for vanishing friction $\gamma_{\mathrm{ex}}=0$, and likewise for vanishing asymmetry parameter $A=0$ (not shown).

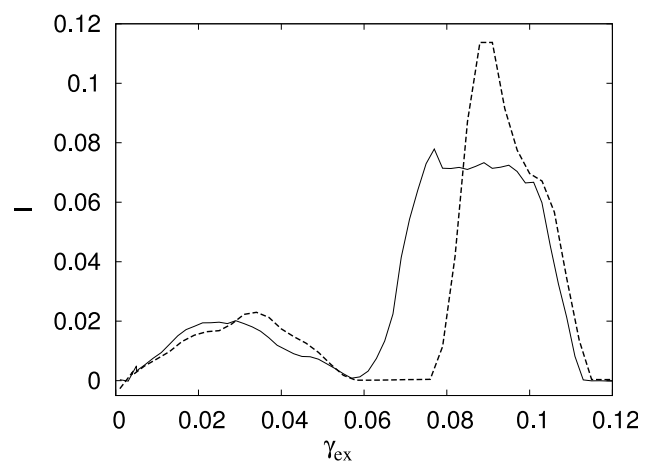

Figure 5: Mean current (7) as a function of the friction coefficient $\gamma_{\mathrm{ex}}$ for the external degree of freedom, for $\varepsilon_{\mathrm{ec}}=7.08$ (full line) and 5.35 (dashed).

As to (ii), in the presence of friction, the existence of persistent transporting trajectories is not guaranteed, in difference to the case of Hamiltonian ratchets where there is always an asymptotic regime of quasi-free motion at high energies [29]. As discussed above, the topology of attractors depends sensitively on couplings, friction parameters, noise strengths etc. In the subsequent paragraphs, we consider in detail the influence of a these parameters on the generation of directed currents in the system.

\subsection{Resonance between chemical and external free- doms}

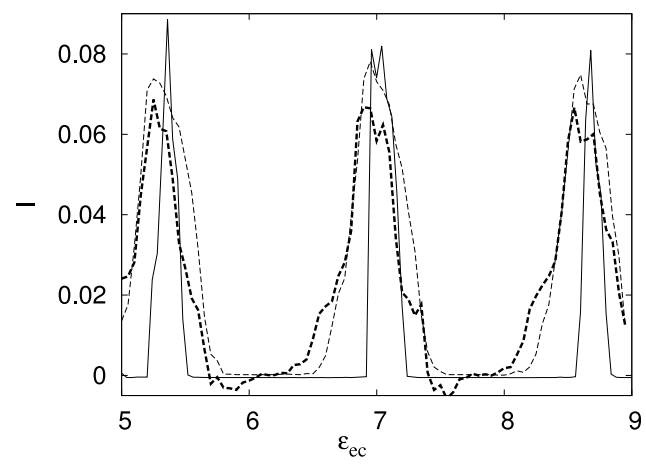

Figure 6: Mean current (7) as a function of the coupling $\varepsilon_{\mathrm{ec}}$ between external and chemical freedom, for different values of the coupling between external and internal freedom, $\varepsilon_{\mathrm{ei}}=$ 0 (full line), 0.5 (bold dashed), 1 (light dashed).

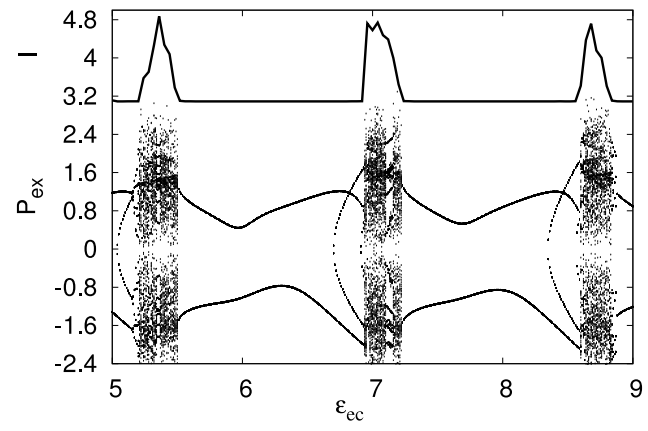

Figure 7: Attractor of the system 6 in terms of the surfaces of section indicated in Figs. 2 and 3 as a function of the coupling $\varepsilon_{\text {ec }}$. Upper part: Mean current (7) (bold dashed) as a function of the same parameter.

In the absence of an external periodic driving, our model is lacking a precise clock that would lead to clearcut resonances. The high inertia of the chemical freedom, however, and the corresponding relatively regular injection of energy "quanta" also defines an approximate time scale that gives rise to resonance-like phenomena. This is obvious from Fig. 6, where marked peaks of the current occur at approximately equidistant values of the externalto-chemical coupling, $\varepsilon_{\mathrm{ec}, n}=n \varepsilon_{\mathrm{ec}, 1}, n=1,2, \ldots$, 
with $\varepsilon_{\mathrm{ec}, 1} \approx 1.8$ in this case. For this value, there is a 1:1 resonance between the two coupled freedoms, i.e., in terms of Fig. 1] the system is moving along diagonal valleys in the two-dimensional array of potential maxima.

However, this resonant behaviour does not amount to simple oscillatory motion in $x_{\mathrm{ex}}$. As is evident from Fig. 7, where we plot a section of the attractor, cf. Fig. 2] vs. $\varepsilon_{\text {ec }}$, the peaks of the current coincide precisely with intervals in $\varepsilon_{\mathrm{ec}}$ where the attractor is fractal. What is more, the sharp definition without shoulders of these peaks can be related to abrupt changes in the attractor structure from limit cycle to chaotic and back.

\subsection{Effects of noise}

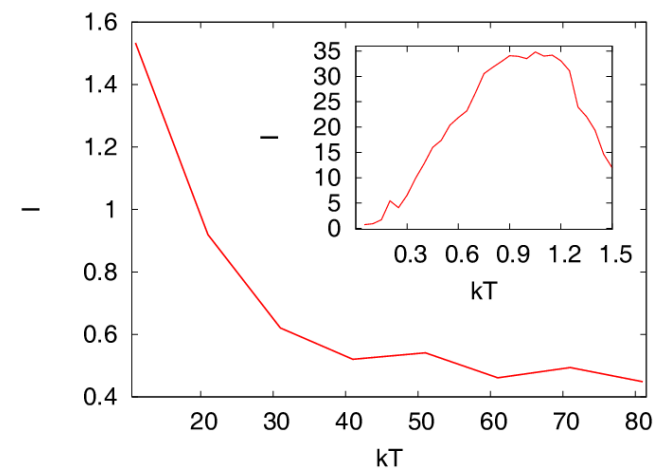

Figure 8: Mean current (7) as a function of the bath temperature $T$. Inset: enlargement of the low-temperature regime $0 \leq T \leq 1.5$.

Figure 8 shows the current vs. noise strength in terms of bath temperature $T$. As would be expected, too strong noise washes out the structure of the attractor, in particular its asymmetry, a necessary condition for directed transport to occur. The inset reveals a more interesting phenomenon: For the other parameter values in question kept constant, there is no extended attractor in the absence of thermal fluctuations, so that the current vanishes at $T=0$. As a consequence, there exists a maximum, albeit broad, of the current at a bath temperature $T \approx 1$, in analogy to stochastic resonance 30 .

\subsection{Rôle of the internal freedom}

A first indication of the influence of the internal freedom can be observed in Fig. 6, comparing the width of the current peaks for different values

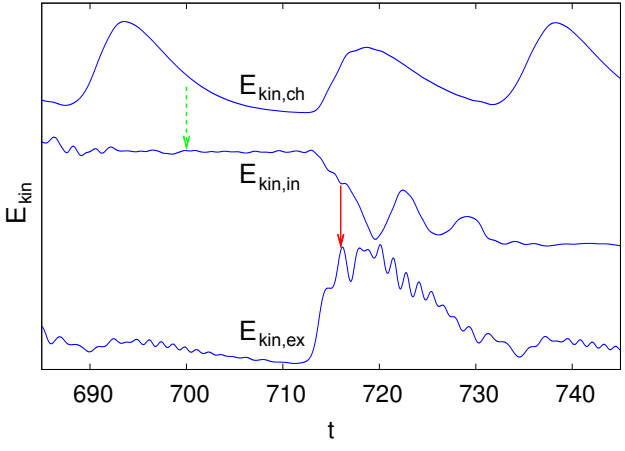

Figure 9: Kinetic energy contained in the chemical (upper graph), internal (middle) and external (lower graph) freedoms as a function of time. The figure shows a particular case. Time dependence is slightly smoothed to suppress irrelevant fluctuations. Arrows indicate typical energy-transfer events from chemical to internal (dashed, green) and from internal to external freedom (full, red). Small-scale oscillations in the lower graph correspond to unit transport steps in $x_{\mathrm{ex}}$. See text.

of the external-internal coupling. They systematically grow broader with increasing $\varepsilon_{\text {ei }}$. This means that off resonance, directed transport is supported or even enabled in the first place by the presence of the internal freedom.

Further insight into the underlying mechanism is provided by Fig. 9, where we plot the kinetic energy in the three respective freedoms as a function of time. It shows a clear tendency for a temporary storage of energy in the internal freedom: The system receives energy almost periodically and in nearly equal portions from the chemical freedom. It tends to accumulate in the internal freedom (green arrow) till a threshold is reached, whereupon it is discharged (red arrow) almost completely to the external freedom, giving rise to a burst of transport steps. This "toilet-flush" behaviour is well-known from neuron firing 31.

Comparing with Fig. 7, we observe moreover that the onset of current with increasing $\varepsilon_{\text {ei }}$ also coincides with a structural change of the attractor from limit cycle to strange. To be sure, two autonomous degrees of freedom are sufficient to allow for chaotic behaviour. Notwithstanding, we can conclude that another relevant rôle of the internal freedom is to induce a richer, more stochastic dynamics of the system. 


\section{Conclusion}

The present work pretends to reduce the gap between abstract models of directed transport and detailed studies of specific motor molecules by constructing a system that endows ratchets with a few essentials of combustion motors, an internal degree of freedom and a coupling to an autonomous chemical freedom as energy source instead of a periodic external force, but ignoring the specifics of particular (classes of) molecules. This allows us to analyze in general terms the rôle of the internal freedom in the generation of transport. We find convincing evidence that it serves as an interface which effectively decouples the output of mechanical work in the form of transport steps from the energy input through hydrolysis or similar reactions. We can observe directly how energy is being stored temporarily in the internal degree of freedom before being channeled to the external freedom. In addition, the internal freedom induces transitions towards a more irregular dynamics of the external freedom, which also contributes to the generation of directed currents.

These results direct attention to processes of energy transfer and dissipation within the molecule, and in particular indicate the functional advantage of a cascaded energy redistribution through intermediate steps 32, refining the simple picture of an external (transport) degree of freedom coupled directly to a structureless bath. Even within the scope of our three-dimensional model, such questions could be studied by changing the configuration of couplings, e.g., coupling the external to the chemical freedom only through the internal one instead of internal-external-chemical as in the present work.

Subtler effects of noisy nonlinear dynamics like stochastic resonance 30] have not been considered in depth. In the context of our model, it is expected to occur in a parameter regime of weak noise close to a resonance of the chemical with the external freedom, cf. Sec. 4.1 lowering the threshold to directed transport as a function of the coupling to the chemical freedom. Quantum effects have not been taken into account, either. We expect relevant modifications of the picture, if any, for the dynamics of the chemical degree of freedom and the injection of chemical energy into functional modes where the discretization of energy and quantum coherence might play a major rôle.

\section{Acknowledgements}

We would like to thank Camilo Aponte, Alfonso Leyva, and José Daniel Muñoz for inspiring discussions and valuable bibliographical information. One of us (TD) gratefully acknowledges financial support by Colciencias and by Volkswagen Foundation during preparation of this work and thanks for the hospitality extended to him by Rensselaer Polytechnic Institute (Troy, NY, USA).

\section{References}

[1] R. D. Astumian, Phys. Rev. Lett. 72 (1994) 1766.

[2] R. Bartussek, P. Hänggi, J. G. Kissner, Europhys. Lett. 28 (1994) 459.

[3] R. Bartussek, P. Reimann, P. Hänggi, Phys. Rev. Lett. 76 (1996) 1166.

[4] R. D. Astumian, Science 276 (1997) 917.

[5] S. Leibler, Nature 370 (1994) 412.

[6] J. A. Spudich, Nature 372 (1994) 515.

[7] W. Wang, O. Donini, C. M. Reyes, P. A. Kollman, Annu. Rev. Biophys. Biomol. Struct. 30 (2001) 211.

8] R. D. Astumian, M. Bier, Biophys. J. 70 (1996) 637.

[9] P. Reimann, Phys. Rep. 361 (2002) 57.

[10] A. L. Tournier, J. C. Smith, Phys. Rev. Lett. 91 (2003) 208106.

[11] S. Nicolay, Y.-H. Sanejouand, Phys. Rev. Lett. 96 (2006) 078104.

[12] J. A. McCammon, B. R. Gelin, M. Karplus, P. Wolynes, Nature 262 (1976) 325.

[13] W. Harrison, Biopolymers 23 (1984) 2943.

[14] B. R. Brooks, M. Karplus, Proc. Natl. Acad. Sci. U.S.A. 82 (1985) 4995.

[15] J. L. Mateos, Physica D 168-169 (2002) 205.

[16] D. Keller, C. Bustamante, Biophys. J. 78 (2000) 541.

[17] K. Kinosita, Cell 93 (1998) 21.

[18] S. Ming, J. Chem. Phys. 118 (2003) 9890

[19] C. A. Aponte, J. D. Muñoz, Biophys. J.

[20] C. S. Peskin, G. Oster, Biophys. J. 68 (1995) 2028.

[21] N. Nakagawa, K. Kaneko, Phys. Rev. E 67 (2003) 040901.

[22] S. Kohler, J. Lehmann, P. Hänggi, Phys. Rep. 406 (2005) 379.

[23] T. Dittrich, N. Naranjo, Rev. Col. Fís. 41 (2009) 440.

[24] S. Flach, Phys. Rev. Lett. 84 (2000) 2358.

[25] F. Reif, Fundamentals of Statistical and Thermal Physics, McGraw-Hill, Boston, 1965.

[26] B. K. Oksendal, Stochastic Differential Equations: An Introduction with Applications, 5th Edition, Springer, New York, 1998.

[27] A. C. Bran'ka, D. M. Heyes, Phys. Rev. E 58 (1998) 2611.

[28] A. C. Bran'ka, D. M. Heyes, Phys. Rev. E 60 (1999) 2381.

[29] H. Schanz, T. Dittrich, R. Ketzmerick, Phys. Rev. E 71 (2005) 026228.

[30] L. Gammaitoni, P. Hänggi, P. Jung, F. Marchesoni, Rev. Mod. Phys. 70 (1998) 223.

[31] A. L. Hodgkin, A. F. Huxley, J. Physiol. 117 (1952) 500.

[32] B. Garraway, Phys. Rev. A 55 (1997) 2290; ibid. 4636. 